\section{Cephalosporin-Gabe trotz allergischer Reaktion}

\author{
Eine Hypersensibilität gegen Cephalosporine betrifft nicht die gesamte \\ Substanzklasse. Daher kann mit geeigneten Vertretern behandelt werden.
}

Da. ass bei einer allergischen Reaktion auf ein bestimmtes Cephalosporin die Behandlung mit anderen Cephalosporinen, die im Pricktest negativ sind, toleriert wird, wurde bislang für einzelne Fälle und kleine Fallserien berichtet. Italienische Allergologen haben Kreuzreaktivität und Verträglichkeit von alternativen Cephalosporinen nun in größerem Maßstab untersucht.

An ihrer Studie beteiligt waren $102 \mathrm{~Pa}$ tienten mit einer Sofortreaktion auf ein Cephalosporin, meistens einer anaphylaktischen Reaktion, und Nachweis einer entsprechenden Sensibilisierung im Pricktest. Sie konnten durch weitere Pricktests, insgesamt auf elf Cephalosporine, sowie durch IgE-Tests für Cefaclor in vier Gruppen eingeteilt werden: Pati- enten der größten Gruppe A $(n=73)$ reagierten positiv auf mindestens einen Wirkstoff aus der Gruppe Ceftriaxon, Cefuroxim, Cefotaxim, Cefepim, Cefodizim und Ceftazidim. Die Reaktionen der Gruppe B $(n=13)$ richteten sich gegen ein Aminocephalosporin (Cefaclor, Cephalexin). Gruppe-C-Patienten ( $n=7)$ waren gegen andere Wirkstoffe als A und B sensibilisiert. In Gruppe D ( $n=9)$ kam es mit Cephalosporinen aus zwei verschiedenen Gruppen zu positiven Reaktionen.

Gegen ein weiteres Cephalosporin aus derselben Gruppe reagierten im Pricktest $45,5 \%$ aus Gruppe A, 26,6\% aus B und $14,3 \%$ aus C. Ein positiver Pricktest mit einem nicht zur Gruppe gehörenden Cephalosporin ergab sich bei 7,6\% der Patienten aus $A, 13,3 \%$ aus $B$ und $0 \%$ aus $C$.
Für die Provokationstests wurden bei Gruppe-A-Patienten Cefaclor, Cefazolin oder Ceftibuten verwendet, bei Gruppe B Cefuroxim, Ceftriaxon, Cefazolin oder Ceftibuten, bei Patienten aus $C$ und $D$ wurde je nach Sensibilisierung aus den genannten Wirkstoffen ausgewählt. Insgesamt wurden 304 Provokationen durchgeführt, alle wurden gut vertragen.

Fazit: Patienten mit einer Cephalosporin-Allergie können, wenn eine Indikation für diese Betalaktame besteht, mit alternativen Cephalosporinen behandelt werden. Die verwendeten Wirkstoffe sollten andere R1-Seitenkettendeterminanten haben als die verantwortliche Substanz und in einem vorgeschalteten Pricktest keine Reaktion auslösen.

Dr. Beate Schumacher

Romano A et al. IgE-mediated hypersensitivity to cephalosporins: Cross-reactivity and tolerability of alternative cephalosporins. J Allergy Clin Immunol 2015; 136: 685-91

\title{
Eisenpräparate mit unterschiedlichem Anaphylaxierisiko
}

\section{Forscher aus den USA haben das Anaphylaxierisiko bei intravenös verabreichten Eisenpräparaten untersucht. Am geringsten ist demnach das Risiko bei Supplementation mit Eisensaccharose.}

U $\mathrm{m}$ retrospektiv das Risiko für eine Anaphylaxie nach intravenöser Anwendung von Eisenpräparaten abschätzen zu können, werteten Wissenschaftler der Food And Drug Administration (FDA) die Daten der staatlichen Krankenversicherung Medicare auf Basis der Einzelleistungsvergütungen aus, die zwischen 2003 und 2013 dokumentiert worden waren. Im Fokus standen dabei Eisendextran, -glukonat und -saccharose sowie Ferumoxytol, Letzeres aber erst ab 2010, also ab dem Zulassungszeitpunkt. Indikationen waren Anämien unter anderem im Zusammenhang mit chronischen Nierenerkrankungen und gastrointestinalen/urogenitalen Blutungen.

Um in die Studie einbezogen werden zu können, durften die Patienten keiner Dialyse unterzogen worden sein und inner- halb des vergangenen Jahres kein intravenös appliziertes Eisenpräparat erhalten haben. Wer für eine Bluttransfusion vorgesehen war oder innerhalb der vergangenen 30 Tage eine anaphylaktische Reaktion hatte, wurde ausgeschlossen.

Insgesamt hatten 274 Patienten nach der erstmaligen Anwendung eines intravenös verabreichten Eisenpräparates eine Anaphylaxie. Bei 170 Patienten war das während einer erneuten Applikation der Fall. Mit jeder Applikation nahm die Rate der Nebenwirkung ab. Das Risiko für eine anaphylaktische Reaktion bei erstmaliger Anwendung lag in der Gruppe der Patienten, die ein Dextranpräparat erhalten hatten, bei 68 pro 100.000 Personen, in der Gruppe der Patienten, die ein nicht dextranhaltiges Präparat erhalten hatten, bei 24 pro 100.000 Personen.
Nach Berücksichtigung mehrerer Parameter wie Alter oder koronare Herzkrankheit errechneten die Wissenschaftler ein 2,6-fach erhöhtes Anaphylaxierisiko im Vergleich zur Anwendung nicht dextranhaltiger Präparate. Im Vergleich zur Applikation von Eisensaccharose war das Anaphylaxierisiko mit Eisendextran (OR: 3,6) und Eisenglukonat (OR: 2,0) ebenfalls erhöht. Ähnliches galt für die Anwendung von Ferumoxytol (OR: 2,2).

Fazit: Das höchste Anaphylaxierisiko bei intravenös verabreichten Eisenpräparaten liegt bei der erstmaligen Anwendung von Eisendextran vor. Am geringsten ist das Risiko bei Supplementation mit Eisensaccharose. Laut den Autoren ist es unklar, warum es zu den anaphylaktischen Reaktionen nach der intravenösen Applikation der Präparate kommt. Diskutiert würden Kreuzreaktionen mit Polysaccharidantikörpern sowie Reaktionen gegen die Kohlenhydrathülle der nicht dextranhaltigen Präparate. Peter Leiner

Wang $C$ et al. Comparative Risk of Anaphylactic Reactions Associated With Intravenous Iron Products. JAMA 2015; 314: 2062-8 